\title{
Remembering Sergio Dain
}

\author{
Abhay Ashtekar ${ }^{1}$ - Roy Maartens ${ }^{2,3}$
}

Published online: 31 May 2016

(C) Springer Science+Business Media New York 2016

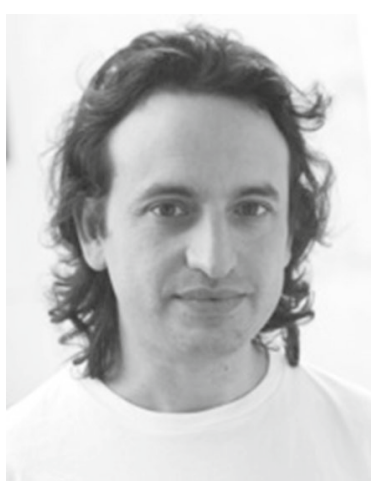

It is with profound sadness that we report on the sudden passing away of Professor Sergio Dain, an Associate Editor of the journal General Relativity and Gravitation. He succumbed to cancer on February 24th, 2016. Although only 46, he had already been an internationally recognized relativist for quite some time.

Sergio received his Ph.D. from the Universidad Nacional de Còrdoba, Argentina in 1999 for his numerous contributions to asymptotically flat space-times and gravitational radiation. Part of his thesis work was carried out at the Max Planck Institute for Gravitational Physics (Albert Einstein Institute-AEI) in Golm, Germany, under the auspices of a Fellowship of the German Academic Exchange Service. In 2000

Abhay Ashtekar

ashtekar@gravity.psu.edu

1 The Pennsylvania State University, University Park, PA, USA

2 University of the Western Cape, Cape Town, South Africa

3 University of Portsmouth, Portsmouth, UK 
he returned to AEI as a post-doctoral research scientist in the Mathematical General Relativity group and remained there till 2007. During this period he made a transition to geometric analysis, specializing in elliptic aspects of Einstein's equations. His very first paper in the area, published as a single author Physical Review Letter, provided a family of initial data representing two spinning black holes. This was followed by a series of papers on conserved quantities in black hole collisions, the close limit approximation, and initial data sets tailored to isolated horizon boundary conditions. He then turned his attention to angular momentum, writing a series of seminal papers establishing inequalities between angular momentum and mass in general relativity. Many of these papers were written after he returned to Cordoba as a faculty member and an independent researcher with CONICET.

These influential contributions were recognized by the International Society on General Relativity and Gravitation through an invitation to give a plenary lecture on the subject during the GR20 and Amaldi10 conference, held at Warsaw in 2013, and by the Fields Institute at Toronto, where he gave two invited lectures on the subject in 2015. He was fully engaged in first rate research until the very end. Indeed, his last paper, establishing sharp bounds on the minimum radius of a charged spherical body in general relativity, appeared in Physical Review D on February 23rd, 2016! It not only contains a hard theorem, but also some numerical simulations and a nice discussion of physical implications-a testimony of Sergio's intellectual breadth.

Sergio was very engaged in the promotion of Science in Argentina at different levels. In particular, he led the Max Planck partner group at the Universidad Nacional de Còrdoba from 2007 to 2012, where he also organized a series of international conferences. Ten students received their graduate degrees under his supervision and he also mentored four post-doctoral researchers. Since January 2014, he served on the Editorial Board of General Relativity and Gravitation. He made important contributions to the journal, remaining active on the Board till the very end. The Board fondly remembers him for his spontaneous sense of humor, his uplifting spirit and his passion for general relativity. The Board and Springer deeply regret that the journal will no longer receive his energetic contributions. We extend our sympathies to his family and many friends. 